\title{
Corticosteroid-binding globulin deficiency
}

INSERM

\section{Source}

INSERM. (1999). Orphanet: an online rare disease and orphan drug data base.

Corticosteroid-binding globulin deficiency. ORPHA:199247

Corticosteroid-binding g lobulin deficiency is a rare, genetic, adrenal disease characterized by diminished corticosteroid-binding capacity associated with normal or low plasma corticosteroid-binding globulin concentration and reduced total plasma cortisol levels. Patients typically present chronic pain, fatigue and hypo/hypertension. 